\title{
APLICAÇÃO DE RESÍDUOS DE CONSTRUÇÃO E DEMOLIÇÃO NA CONSTRUÇÃO CIVIL: UMA REVISÃO BIBLIOGRÁFICA SISTEMÁTICA
}

\author{
MENEGHETTI, MAURÍCIO \\ Engenheiro Civil \\ PUCRS \\ Rio Grande do Sul; Brasil \\ mauricio.meneghetti@acad.pucrs.br
}

\author{
ANDRADE, JAIRO \\ Professor \\ PUCRS \\ Rio Grande do Sul; Brasil \\ jairo.andrade@pucrs.br
}

\section{RESUMO}

As questões de sustentabilidade são uma das principais preocupações nos dias de hoje. A reutilização de entulhos na indústria da construção civil é uma fonte alternativa de materiais de construção que pode substituir o uso de materiais virgens. Reduzindo-se assim o impacto ambiental e o volume de resíduos despejados em aterros sanitários e de forma irregular. O objetivo deste estudo foi verificar, através de uma revisão bibliográfica sistemática, as publicações relacionadas à aplicação de resíduos de construção e demolição na própria construção civil. Foram encontrados 127 artigos relacionados ao tema proposto, entre os anos 2007 e 2017, chegando-se as principais tendências de pesquisa. Os principais materiais estudados foram concreto, tijolos, argamassa e cerâmicas. E as aplicações mais adotadas foram para a fabricação de concreto, argamassa e para o uso na área de pavimentação.

Palavras-chave: Resíduos de construção e demolição, Revisão bibliográfica sistemática, Reciclagem e Reuso.

\section{ABSTRACT}

Sustainability issues are a major concern today. The reuse of wastes in the construction industry is an alternative source of building materials that can replace the use of raw materials. This reduces the environmental impact and the volume of waste dumped in landfills and in an irregular way. The objective of this study was to verify, through a systematic bibliographic review, the publications related to the application of construction and demolition waste in civil construction itself. Were found 127 articles related to the proposed theme, between the years 2007 and 2017, reaching the main research trends. The main materials studied were concrete, bricks, mortar, and ceramics and the most adopted applications were for the manufacture of concrete, mortar and use in the paving area.

Keywords: Construction and demolition waste, systematic bibliographic review, Recycling.

\section{INTRODUÇÃO}

A construção civil é uma das atividades mais antigas desenvolvidas pela humanidade e desde seus primórdios foi executada de forma artesanal, gerando grandes quantidades de entulho mineral como subproduto. Na época do Império Romano tal fato já despertou atenção dos construtores e deste período datam os primeiros registros de reutilização de resíduos da construção na produção de novas obras. Porém, a primeira aplicação significativa de entulho reciclado só foi registrada após o término da $2^{\circ}$ Guerra Mundial, na reconstrução das cidades europeias, onde escombros e entulhos resultantes de edificações totalmente demolidas foram usados na produção de agregados, visando atender a demanda da época. Então se pode dizer que a partir de 1946 teve início o desenvolvimento da tecnologia de reciclagem de resíduos da construção civil (RCC) (LEVY, 1995).

Segundo Santos (2012), a indústria da construção civil tem grande importância para o desenvolvimento econômico e social, mas ainda causa muitos impactos negativos do ponto de vista ambiental. De acordo com Mesquita (2012), a cadeia produtiva da construção civil, além do excessivo emprego de energia, água e terra, consome entre 20 e $50 \%$ dos recursos naturais de todo planeta. A indústria construção civil também gera impactos no meio ambiente com a produção de resíduos, que é uma atual problemática das grandes cidades. Estima-se que o entulho possa chegar a $60 \%$ dos resíduos sólidos urbanos produzidos e aproximadamente a metade destes são dispostos de forma irregular. Então se conclui que 
por tratar-se de um tema de grande impacto à economia, sociedade e ao meio ambiente, fica evidenciada a importância do estudo do reaproveitamento dos rejeitos de construção e demolição.

A questão dos resíduos é historicamente um problema para a sociedade, uma vez que o homem nunca encontrou uma solução realmente efetiva. Desta forma, as primeiras ações para enfrentar este problema surgiram na década de 1980 na Europa, enquanto que no Brasil, somente no início do século XXI foram tomadas medidas para enfrentar estas adversidades. Apenas em julho de 2002, quando houve a aprovação de Resolução de n³07 pelo Conselho Nacional do Meio Ambiente (CONAMA), foram estabelecidas diretrizes, critérios e procedimentos para a gestão de resíduos da construção civil (BRASILEIRO; MATOS, 2015).

A busca de uma solução para os rejeitos da construção civil é um assunto de grande importância na atualidade. Por este motivo nota-se o interesse por pesquisas cientificas sobre este conteúdo. Dentre as diversas formas disponíveis para a realização de uma pesquisa científica tem-se o emprego da Revisão Bibliográfica Sistemática (RBS). De acordo com Galvão e Pereira (2014) a atividade de compilar dados científicos sobre um assunto é praticado há muito tempo. Porem só a partir da década de 1950 surgiu às revisões denominadas de sistemáticas.

Segundo Galvão e Pereira (2014), ao se estudar um tema, frequentemente encontra-se resultados contraditórios. Para tentar esclarecer estas controvérsias deve-se apoiar-se nas melhores publicações sobre o assunto e, baseado nestes princípios, surgiu a RBS. De maneira genérica, tal técnica consiste na investigação focada em uma questão bem definida, que visa identificar, selecionar e coletar os estudos publicados sobre uma temática para a posterior análise e síntese das evidências geradas.

Desta forma, tem-se como objetivo geral do presente trabalho fazer, através da RBS, um levantamento identificando, selecionando e avaliando as publicações que abordam como tema de estudo a reutilização de resíduos de construção e demolição na própria construção civil. Como objetivos específicos podem ser citados: (i) verificar a quantidade de publicações associadas com o tema RCD; (ii) coletar e analisar tendências de estudos e conclusões.

Em um estudo desta natureza algumas delimitações se fazem necessárias. Desta forma não serão analisadas teses e dissertações. Serão verificados artigos publicados nas bases de dados selecionas para a RBS e com sua data de publicação entre os anos de 2007 a 2017.

\section{PROCEDIMENTOS METODOLÓGICOS}

\subsection{Planejamento da RBS}

Na etapa de planejamento da RBS primeiramente foi registrado o objetivo da revisão: identificar as publicações que abordam como tema de estudo a reutilização de resíduos de construção e demolição na própria construção civil. A busca teve como foco artigos que tratassem de aspectos técnicos, sendo assim excluídos, por exemplo, artigos de aspectos ligados à gestão e às legislações. Portanto as perguntas que motivaram o trabalho e deveriam ser respondidas através da análise dos artigos foram:

- Há o estudo de reutilização de RCD na construção civil?;

- Quais são os principais resíduos que estão sendo estudados?;

- Onde estão sendo empregados estes resíduos?;

Na sequência foi realizado o desenvolvimento do protocolo de pesquisa. Para o método realizou-se uma busca nas principais bases de dados eletrônicas utilizadas na área da pesquisa das Engenharias, cujas selecionadas como fonte de busca foram:

- Science Direct: website operado por uma editora de literatura científica com acesso a mais 12 milhões de publicações e mais de 3800 revistas científicas, o site é uma importante ferramenta de pesquisa de diversas áreas (SCIENCE DIRECT, 2017);

- Scopus: é o maior banco de dados de citações e resumo de literatura revisada por pares: revistas científicas, livros e conferências. Apresenta ferramentas inteligentes para rastrear, analisar e visualizar a pesquisa (SCOPUS, 2017)

- Periódicos CAPES: biblioteca virtual da Coordenação de Aperfeiçoamento de Pessoal de Nível Superior (CAPES). Reúne e disponibiliza artigos periódicos nacionais e internacionais, é considerado uma importante fonte para as atividades de pesquisa e ensino no Brasil (CAPES, 2017). 
A RBS baseou-se nos idiomas inglês, por ser a língua internacional e a mais utilizada para a escrita de artigos científicos, e o português, por ser a língua primaria do pesquisador e do país de publicação do artigo. Adotou-se as palavras chaves "construction and demolition waste", "recycling" and "reuse" para a busca dos artigos. Os artigos pesquisados foram analisados de acordo com os seguintes critérios de inclusão e exclusão:

- Acessibilidade de arquivos não restrita;

- Seleção de publicações que possuam no título ou nas palavras chaves os assuntos de busca definidos previamente;

- Documento do tipo: article ou review;

- $\quad$ Artigos publicados entre os anos de 2007 e 2017.

\subsection{Realização da RBS}

O processo de realização desta revisão (Figura 1) iniciou-se através da busca nas bases de dados selecionadas. As publicações verificadas compreendiam-se entre o período de 2007 até maio de 2017, a fim de mostrar a situação dos estudos mais recentes. A seleção abrangeu artigos publicados em periódicos científicos indexados, congressos ou conferências.

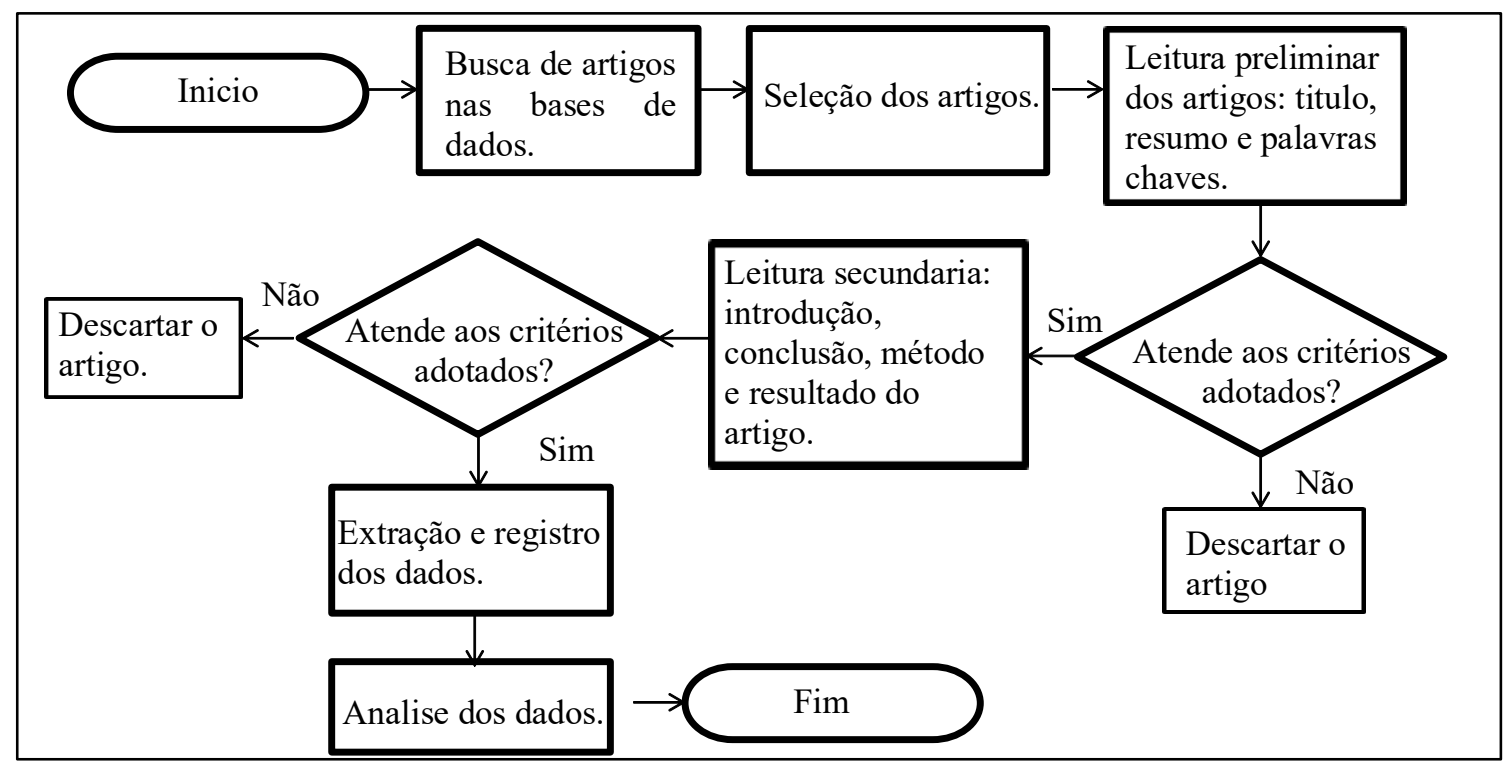

Figura 1: Fluxograma da etapa de Realização da RBS

Cada um dos artigos foi analisado de acordo com os objetivos identificados na etapa de planejamento da RBS. Na seleção preliminar, os artigos encontrados após a busca tiveram como foco de leitura o título, as palavras chaves e o resumo. Nesta leitura verificaram-se as pesquisas de modo a assegurar-se que estavam de acordo com os critérios de inclusão e exclusão apresentados no protocolo de busca.

Na seleção secundária foi iniciada a leitura da introdução e conclusão dos artigos, seguido pelos métodos e resultados encontrados. Fazendo-se, portanto, a exclusão das publicações que não possuíam o tema reutilização de resíduos de construção e demolição na construção civil em evidência e não respondessem as perguntas que motivaram o trabalho (Há o estudo de reutilização de RCD na construção civil?; Quais são os principais resíduos que estão sendo estudados? Onde estão sendo empregados estes resíduos). Após o processo de leitura, extração, registro e análise dos dados, finalizou-se o processo de busca, conforme apresentado na tabela .

Tabela 1: Resultados das buscas

\begin{tabular}{l|c|c|c}
\hline Base de dados & Publicações encontradas & Seleção preliminar & Seleção secundária \\
\hline Science Direct & 2556 & 41 & 19 \\
\hline Scopus & 1511 & 273 & 96 \\
\hline Periódicos CAPES & 5717 & 103 & 26 \\
\hline
\end{tabular}




\begin{tabular}{l|l|l|l}
\hline Total & 9784 & 417 & 141 \\
\hline
\end{tabular}

Dentre os 141 artigos filtrados após a seleção secundaria verificou-se que 14 repetiam-se. Portanto, 127 documentos avançaram para a fase de extração e registro das informações. Foi utilizada uma planilha elaborada em Excel com os seguintes dados: título, palavras chave, autores, ano de publicação, país de origem, nome da base de dados onde foi encontrado, os tipos de RCD estudados e onde estes RCD estão sendo aplicados. Assim, foi possível realizar a análise de conteúdo dos trabalhos, desde dados mais simples, até uma análise mais aprofundada, como a abordagem da pesquisa.

A tabela 72 apresenta o ranking de fator de impacto das revistas cientificas selecionadas na RBS. O fator de impacto é uma medida da frequência com que o artigo, de um determinado periódico, foi citado em um delimitado ano ou período (Scimago Journal \& Country Rank, 2017). Os periódicos com maiores fatores de impacto são frequentemente considerados mais importantes do que aqueles com os valores mais baixos.

Tabela 2: Fator de impacto das revistas cientificas.

\begin{tabular}{c|l|c|c}
\hline Posição & \multicolumn{1}{|c|}{ Nome do periódico } & Fator de impacto & Número de artigos \\
\hline $1^{\circ}$ & Composites Part B: Engineering & 4,727 & 1 \\
\hline $2^{\circ}$ & Cement and Concrete Composites & 4,265 & 5 \\
\hline $3^{\circ}$ & Waste Management & 4,03 & 3 \\
\hline $4^{\circ}$ & Materials and Design & 3,83 & 2 \\
\hline $5^{\circ}$ & Resources, Conservation and Recycling & 3,313 & 3 \\
\hline $6^{\circ}$ & Construction and Building Materials & 3,169 & 67 \\
\hline $7^{\circ}$ & Advances in Materials Science and Engineering & 1,299 & 1 \\
\hline $8^{\circ}$ & Materials Research & 1,01 & 1 \\
\hline $9^{\circ}$ & Materiales de Construccion & 0,96 & 1 \\
\hline $10^{\circ}$ & Procedia Engineering & 0,73 & 10 \\
\hline $11^{\circ}$ & Journal of Advanced Concrete Technology & 0,658 & 21 \\
\hline $12^{\circ}$ & Physics Procedia & 0,64 & 1 \\
\hline $13^{\circ}$ & Transportation Research Procedia & 0,61 & 1 \\
\hline $14^{\circ}$ & Ceramics - Silikaty & 0,485 & 1 \\
\hline $15^{\circ}$ & Periodica Polytechnica: Civil Engineering & 0,356 & \\
\hline $16^{\circ}$ & Acta Scientiarum - Technology & 0,315 & 1 \\
\hline $17^{\circ}$ & Proceedings Of The Institution Of Civil Engineers & 0,197 & 1 \\
\hline $18^{\circ}$ & Agriculture and Agricultural Science Procedia & 0,135 & 1 \\
\hline $19^{\circ}$ & Procedia - Social and Behavioral Sciences & - & 1 \\
\hline $20^{\circ}$ & International Journal of Sustainable Built Environment & - & 1 \\
\hline $21^{\circ}$ & HBRC Journal & - & 1 \\
\hline
\end{tabular}

\section{RESULTADOS E DISCUSSÃO}

Os dados extraídos foram analisados buscando identificar as práticas de acordo com os objetivos propostos, com eles foram gerados gráficos e tabelas a fim de uma melhor visualização facilitando a interpretação das informações encontradas e, assim, tomar conhecimento da situação mundial das aplicações dos RCD na construção civil. A figura 2 apresenta o ano de publicação dos artigos analisados.

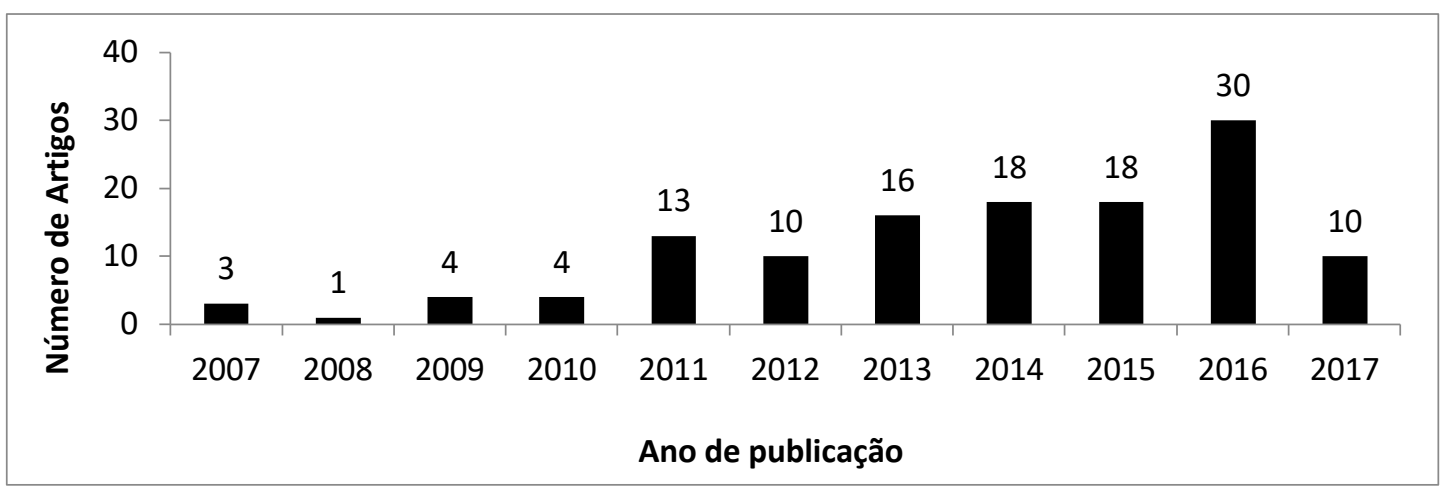

Figura 2: Distribuição anual das publicações estudadas. 
Pode-se observar uma tendência de crescimento de estudos na temática de reutilização dos entulhos da construção civil. Porém, a partir do ano de 2011 onde houve um grande crescimento no numero de publicações, fica-se evidenciado o aumento de interesse dos pesquisadores pelo assunto. Na Figura 3 encontram-se apresentados os principais países envolvidos nas pesquisas.

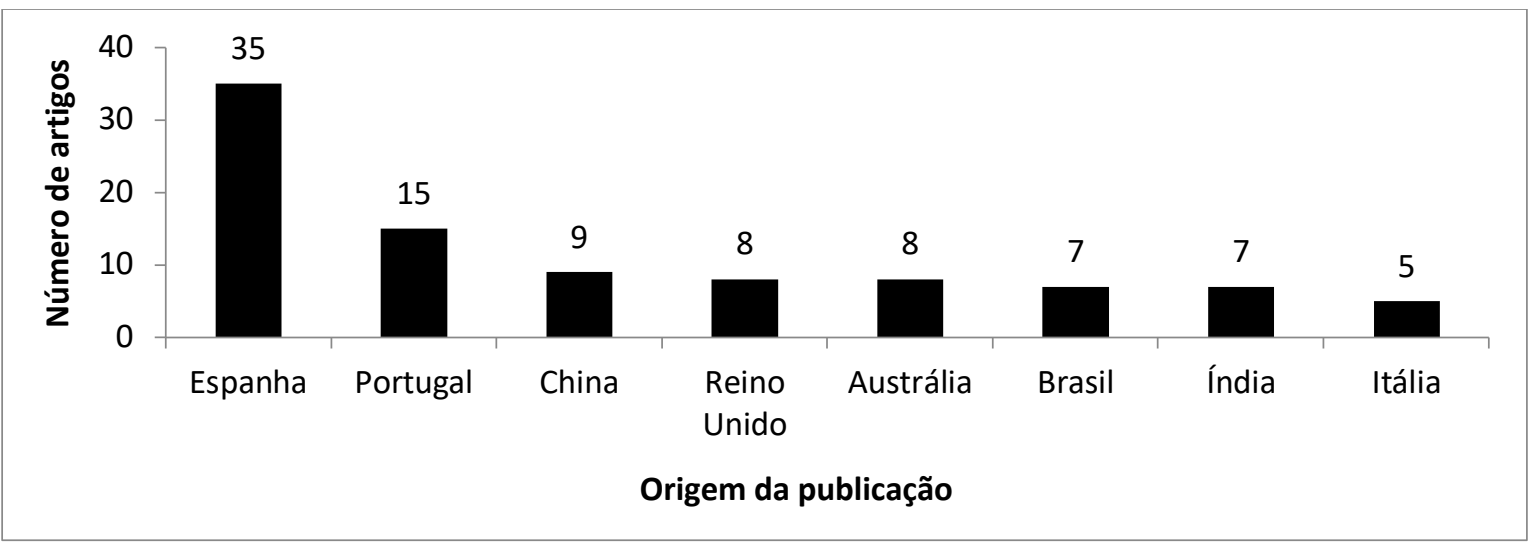

Figura 3: Distribuição por países das publicações estudadas.

Dos 127 artigos analisados na RBS foram identificadas publicações de 28 diferentes nacionalidades. Por esta diversidade de nações é possível perceber que a questão de reaproveitamento dos RCD é uma preocupação mundial, pois países de diversos continentes e diferentes níveis de desenvolvimento apresentam publicações referentes ao assunto.

O total de pesquisas relacionadas à Espanha e Portugal, ocorre pelo fato de que até o ano de 2008 a reciclagem e o reuso de RCD nestes países não chegava a 10\% da quantidade gerada. Porém, na Espanha a legislação para controle de produção e gestão de RCD foi publicada em 2008. Em Portugal, também em 2008, foi aprovada a regulamentação do fluxo de RCD com a finalidade de reprimir as grandes quantidades geradas e deposições ilegais desses resíduos (GEUS e GARCIA, 2014). Consequentemente, após 2008 necessitou-se uma busca por maior conhecimento de gestão de resíduos por parte dos pesquisadores destes países, gerando assim um maior volume de artigos publicados, conforme identificado na figura 3.

Os RCD são compostos por uma grande variedade de elementos, onde os materiais cujo reuso e a reciclagem são mais estudados estão identificados na figura 4. Verificou-se que alguns artigos abrangem o estudo de mais de tipo de material, salientando-se também que houve artigos onde os materiais não foram descriminados e classificaram-se de forma genérica como RCD.

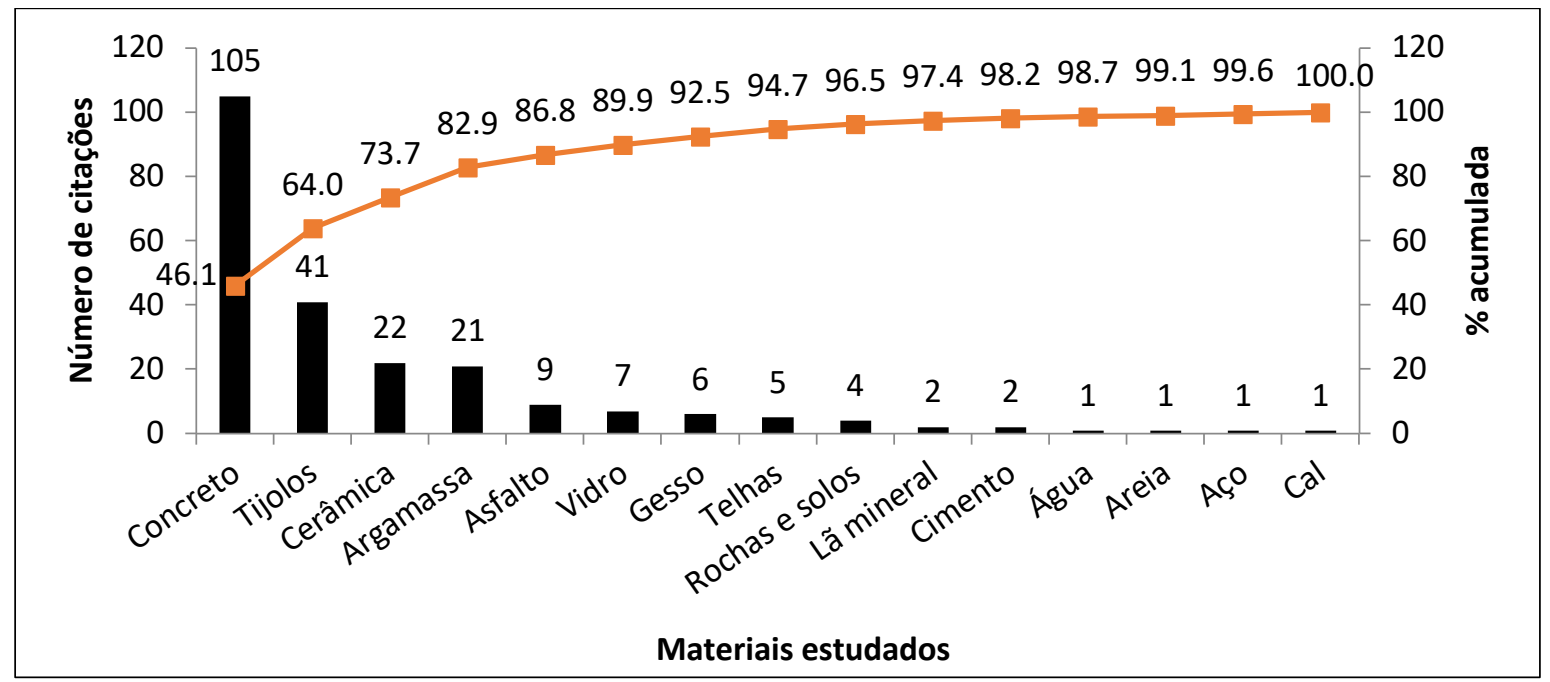

Figura 4: Materiais estudados para a reciclagem e reuso. 
Pode-se verificar que o concreto, os tijolos, as cerâmicas e a argamassa são os materiais mais citados nas publicações. Então, de acordo com a figura 4, conclui-se que os resíduos mais analisados para o reuso e reciclagem, são também os mais gerados na indústria da construção civil. O emprego destes rejeitos encontra-se apresentado na figura 5.

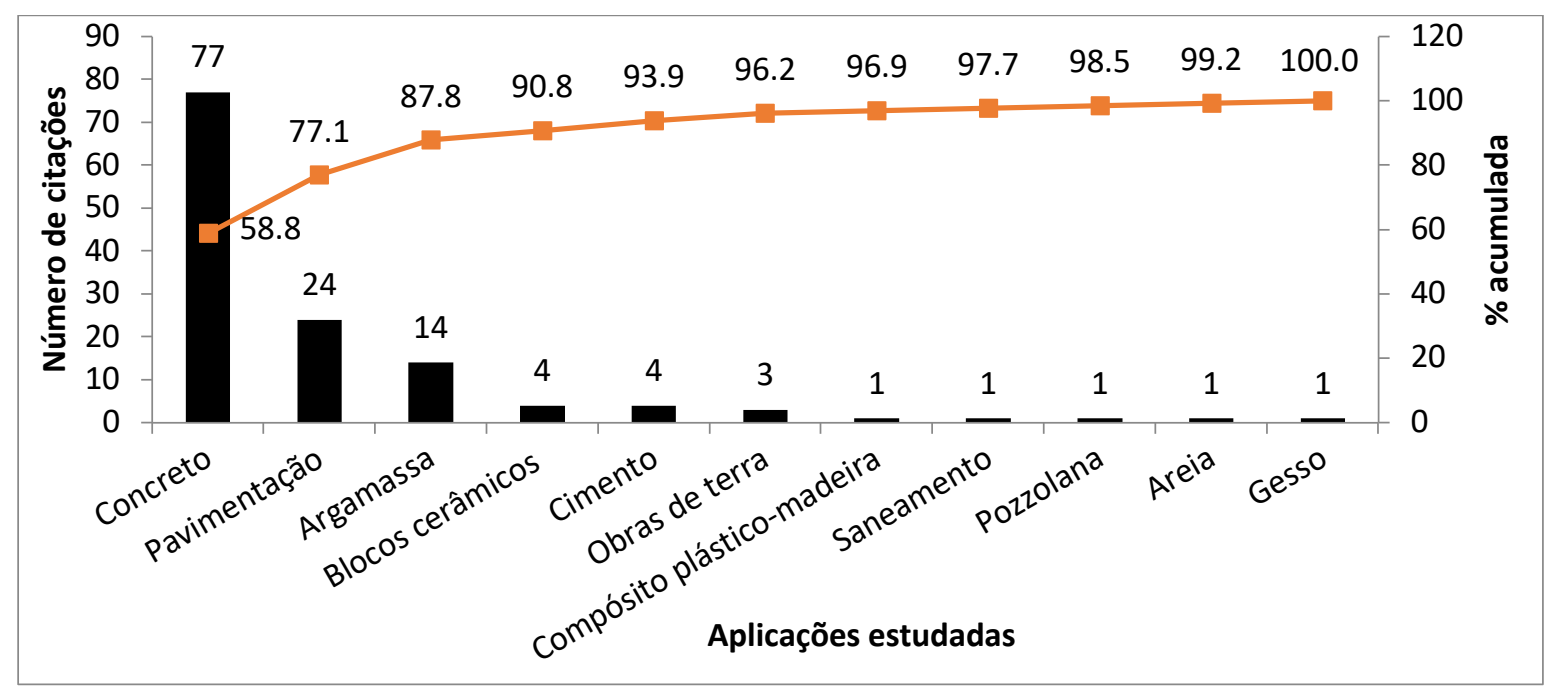

Figura 5: Aplicação dos resíduos RCD.

Foram encontrados artigos que abordam mais de uma prática de utilização dos RCD. O estudo de RCD para fabricação de concreto totalizou 77 citações nas pesquisas selecionadas. O que representa aproximadamente $59 \%$ de todas as práticas adotadas; as aplicações em pavimentação giram entorno de $18 \%$ e para argamassa $11 \%$, respectivamente. Ao finalizar-se a analise da RBS observaram-se algumas tendências de pesquisas, conforme se pode verificar na tabela 3.

Tabela 3: Tendências de pesquisas relacionadas ao reuso e reciclagem de RCD.

\begin{tabular}{l} 
Descrição das pesquisas \\
\hline Os artigos investigaram as propriedades mecânicas, químicas, físicas e de \\
durabilidade de concretos confeccionados com RCD. Buscando-se \\
proporções ótimas de substituição de agregados naturais por agregados \\
reciclados, onde se atendesse aos requisitos exigidos por normas \\
internacionais.
\end{tabular}

Os trabalhos tiveram um maior enfoque no estudo das características físicas e mecânicas dos agregados reciclados. Na sequência procurou-se identificar a influência da composição e da qualidade dos resíduos nos atributos dos concretos reciclados.

Foram também identificadas publicações que trataram da viabilidade do uso de RCD para a utilização em peças de concreto pré-fabricadas. As principais aplicações foram para a confecção de blocos de concreto, meios-fios de estradas, pisos e tubos de concreto.

Realizaram-se estudos laboratoriais sobre a possibilidade de projetar misturas de asfalto através de resíduos de construção e demolição, para uso na pavimentação de estradas. Realizaram-se ensaios de propriedades mecânicas como a rigidez, deformação permanente e fadiga.

\begin{tabular}{l}
\multicolumn{1}{c}{ Autores } \\
(MEDINA et al., 2015) \\
(AWOYERA et al., 2016) \\
(MORICONI; TARELA, 2017) \\
(LÓPEZ-GAYARRE et al., 2011) \\
(DOMINGO-CABO et al., 2009) \\
(KOU; ZHAN; POON, 2012) \\
(TABSH; ABDELFATAH, 2009) \\
(WAGIH et al., 2013) \\
(CABRAL et al., 2012) \\
(SILVA; DE BRITO; DHIR, 2014) \\
(KOPER; KOPER; KOPER, 2017) \\
(SARAVANAKUMAR; ABHIRAM; \\
MANOJ, 2016) \\
(AGRELA et al., 2011) \\
(SOUTSOS; TANG; MILLARD, 2011) \\
(MARTÍN-MORALES et al., 2017) \\
(ÖZALP et al., 2016) \\
(LÓPEZ GAYARRE et al., 2013) \\
(SOUTSOS; TANG; MILLARD, 2012) \\
(SABAI et al., 2013) \\
(PASANDÍN; PÉREZ, 2014) \\
(PASANDÍN; PÉREZ, 2013) \\
(GÓMEZ-MEIJIDE et al., 2015) \\
(GÓMEZ-MEIJIDE; \\
PASANDÍN, 2016) \\
(PÉREZ; PASANDÍN; MEDINA, 2012) \\
\hline
\end{tabular}




\begin{tabular}{|c|c|}
\hline $\begin{array}{l}\text { Apresentaram-se ensaios de caracterização mecânica de agregados } \\
\text { granulares reciclados passa utilização como camadas de base e sub-base } \\
\text { de estradas. A influência da variabilidade de mateiras e o efeito do } \\
\text { tratamento com cimento destas misturas também foi estudado. }\end{array}$ & $\begin{array}{l}\text { (ARULRAJAH et al., 2014) } \\
\text { (ARULRAJAH et al., 2016) } \\
\text { (ARISHA et al., 2016) } \\
\text { (XUAN; MOLENAAR; HOUBEN, 2015) } \\
\text { (XUAN et al., 2014) } \\
\text { (XUAN; MOLENAAR; HOUBEN, 2016) } \\
\text { (CERNI; CARDONE; BOCCI, 2012) }\end{array}$ \\
\hline $\begin{array}{l}\text { Estas pesquisas tiveram como objetivo estudar a viabilidade de } \\
\text { incorporação de fração fina de agregados reciclados provenientes de } \\
\text { resíduos de construção e demolição na fabricação de argamassas. Foram } \\
\text { utilizadas diferentes proporções de cimento, agregados e diferentes } \\
\text { porcentagens de substituição agregados naturais por agregados reciclados. } \\
\text { Após avaliou-se o comportamento mecânico e reológico das amostras. }\end{array}$ & $\begin{array}{l}\text { (MARTÍNEZ et al., 2016) } \\
\text { (CORINALDESI; MORICONI, 2009) } \\
\text { (CUENCA-MOYANO et al., 2014) } \\
\text { (CABRERA-COVARRUBIAS et al., } \\
\text { 2017) } \\
\text { (JIMÉNEZ et al., 2013) } \\
\text { (OLIVEIRA; DE BRITO; VEIGA, 2015) }\end{array}$ \\
\hline
\end{tabular}

Para concreto verificou-se uma grande quantidade de pesquisas de substituição de agregados naturais (AN) por agregados reciclados (AR), chegando-se a proporções ótimas onde o concreto reciclado mantinha as propriedades mecânicas e durabilidade semelhantes ao concreto de agregado natural. As resistências à compressão e à ruptura do concreto feito com AR dependem das proporções da mistura. Em geral, para mesmas relações a/c, a resistência do concreto reciclado pode ser 10-25\% menor que a do concreto convencional (TABSH; ABDELFATAH, 2009) e (BEHERA et al., 2014). Os motivos que podem gerar estas perdas são devidos à qualidade do $\mathrm{AR}$, aumento da porosidade do concreto, ligação interfacial fraca entre o agregado e a pasta de cimento e a presença de microfissuras e fissuras que podem reduzir a ligação com a pasta de cimento circundante.

Elchalakani, Elgaali, (2012) tambem observou reduções de resistência entre 12-25\% para concretos reciclados com $100 \%$ de substituição, porem recomenda-se razoes de ate $80 \%$ de substituição de AN por AR, onde se obteve resultados muito próximos ao concreto com agregado natural. Os ensaios mostram que as propriedades mecânicas do concreto reciclado feito de agregados reciclados dependem, sobretudo, da qualidade do material reciclado e não da sua quantidade. Em geral, a qualidade do material reciclado obtido a partir dos resíduos de construção foi melhor que a qualidade do material obtido a partir dos resíduos de demolição.

Os resultados confirmam que a utilização de RCD para construção de concreto é bastante viável, uma vez que a resistência à compressão, durabilidade e o módulo de elasticidade ainda atingem valores consideráveis. No entanto, estas informações devem ser usadas com cautela, e testes experimentais devem sempre ser realizados para certificar propriedades de concreto (CABRAL et al., 2012). Além disto, verificou-se que o concreto reciclado usando o método de substituição de agregados pode adquirir qualidade suficiente como concreto estrutural e para produtos pré-fabricados (DOSHO, 2007).

A área de pavimentação encontrou-se subdivida em duas categorias: uso de RCD para a confecção de asfaltos ou para o emprego em camadas de base e sub-base de estradas. No que se diz respeito a asfalto foram estudados principalmente as propriedades mecânicas (como rigidez, deformação e fadiga). Entre outras conclusões, os resultados mostraram que, embora as misturas de asfalto contendo RCD apresentem uma maior perda de água durante o processo de cura, pode-se obter valores de rigidez superiores àquelas das misturas de controle usando agregado natural (GÓMEZ-MEIJIDE et al., 2015), (GÓMEZ-MEIJIDE; PÉREZ; PASANDÍN, 2016), (PÉREZ; PASANDÍN; MEDINA, 2012). As misturas AR com diversos teores de substituição $(5 \%, 10 \%, 20 \%, 30 \%)$ do AN para aplicação como asfalto apresentaram boas propriedades de engenharia e cumprem as especificações internacionais (PASANDÍN; PÉREZ, 2013).

Quanto a camadas de base e sub-bases de estradas, as pesquisas tratam do assunto como alternativa sustentável e efetiva aos agregados tradicionais. Com base na avaliação das características como a resistência ao cisalhamento, verificou-se que os agregados reciclados compactados têm o potencial de ser utilizados em aplicações de base grosseira e camada subbase para estradas (ARULRAJAH et al., 2014), (CERNI; CARDONE; BOCCI, 2012), (LEITE et al., 2011). Com ensaios experimentais ainda foi possível alcançar melhores resultados quando os materiais foram tratados com cimento (XUAN; MOLENAAR; HOUBEN, 2015), (ARULRAJAH et al., 2016). O tratamento dos entulhos com cimento proporcinou um aumento da resistência à tração e compressão dos materiais, e também contribuiu para uma maior capacidade de distribuição das cargas nas camadas de base e sub-base estudadas. 
A aplicação de RCD para confecção de argamassas foi um assunto bastante estudado. De acordo com os resultados do estudo de dosagens, as argamassas recicladas apresentam comportamento de resistência mecanica pior do que as argamassas fabricadas usando suporte natural, no entanto, cumprem os limites estabelecidos pelas normas e fabricantes (SAIZ MARTÍNEZ et al., 2016). Os resultados experimentais mostraram que a resistência mecânica das argamassas preparadas com RCD é significativamente inferior à referência. No entanto, a resistência de ligação na interface entre argamassa e tijolo resultou ser maior para argamassas preparadas com agregados reciclados (SILVA; DE BRITO; DHIR, 2016), (CORINALDESI; MORICONI, 2009). Os resultados sugerem que, à medida que o conteúdo de $A R$ aumenta, a força de adesão também aumenta. Tendo em mente que a argamassa reciclada demonstra maior retenção de água, é possível que isso evite uma seca rápida indesejada da argamassa e também uma grande quantidade de água absorvida pelo tijolo, levando a uma hidratação mais efetiva da pasta de cimento e maior resistência adesiva ao substrato.

Razões de substituição de até $40 \%$ em volume não afetaram significativamente as propriedades da argamassa fresca e dura, com exceção da densidade e da trabalhabilidade. Portanto, o uso de agregados finos reciclados na fabricação de argamassa de alvenaria poderia ser uma alternativa viável que ajudaria a aumentar a taxa de reciclagem de resíduos de construção e demolição e apoiar o desenvolvimento sustentável no setor de construção (JIMÉNEZ et al., 2013).

\section{CONSIDERAÇÕES FINAIS}

Este estudo apresenta uma revisão bibliográfica sistemática, cujo objetivo é analisar os artigos que abordam como tema de estudo a reutilização de resíduos de construção e demolição na própria construção civil. A pesquisa não tem a aspiração de apresentar todas as práticas, visto que a mesma é dependente do enquadramento das publicações encontradas nos critérios específicos definidos no protocolo de seleção dos dados. Mesmo com tal limitação, é creditado que este artigo possua conteúdo suficiente para desenvolver conclusões.

Com base na RBS apresentada, após uma análise das publicações no período de 2007 a 2017 identificou-se um elevado numero de artigos sobre o tema. Existe uma clara tendência de crescimento de interesse neste assunto por parte dos pesquisadores, visto que com a expansão dos centros urbanos a gestão dos resíduos se tora um problema cada vez maior. Também se concluiu que este objeto de estudo não é alvo apenas de países desenvolvidos e com capacidade tecnológica como Espanha, Austrália, Estados Unidos e Japão. Países em desenvolvimento e com menos tecnologias como China, Brasil, Índia, Egito e México igualmente estudam este problema.

No presente artigo constatou-se que os RCD mais citados nas pesquisas para a reaplicação na construção civil são o concreto, tijolos, cerâmicas e argamassa. Portanto é possível associar que os materiais mais estudados são também os mais utilizados na construção civil, que consequentemente geram o maior volume de entulhos após a demolição de obras. As principais vertentes de pesquisas da aplicação dos RCD na indústria da construção civil se dá na fabricação de concretos, argamassas e o uso em pavimentação de estradas. Através da análise dos artigos selecionados, os resultados apresentados pelos autores expressam que, mesmo com as delimitações, a reutilização de resíduos de construção e demolição na própria construção civil é viável e uma boa alternativa para diminuir o impacto ambiental gerado pela indústria da construção civil.

\section{REFERÊNCIAS}

ARISHA, A. et al. Using Blends of Construction \& Demolition Waste Materials and Recycled Clay Masonry Brick in Pavement. Procedia Engineering, v. 143, n. Ictg, p. 1317-1324, 2016.

BIOLCHINI, J.; MIAN, P.G.; NATALLI, A.C.C.; TRAVASSOS, G.H. (2005). Systematic Review in Software Engineering. Technical Report RT-ES 679/05, COPPE/UFRJ.

BRASIL. Ministério do Meio Ambiente. Resolução Conama no 307, de 5 de julho de 2002. Estabelece diretrizes, critérios e procedimentos para a gestão dos resíduos da construção civil. Diário Oficial da União, Brasília, 17 jul. 2002.

BRASIL. Ministério do Meio Ambiente. Resolução Conama no 358, de 29 de abril de 2005. Dispõe sobre o tratamento e a disposição final dos resíduos dos serviços de saúde e dá outras providências. Diário Oficial da União, Brasília, 4 maio 2005 .

BRASILEIRO, L. L.; MATOS, J. M. E. Revisão bibliográfica: reutilização de resíduos da construção e demolição na indústria da construção civil. Cerâmica, v. 61, n. 358, p. 178-189, 2015. 
CONTRERAS, M. et al. Recycling of construction and demolition waste for producing new construction material (Brazil case-study). Construction and Building Materials, v. 123, p. 594-600, 2016.

CORREAA, L. R. Sustentabilidade na Construção Civil. p. 1-70, 2009.

CUDNEY, E. A.; GILLIS, W. L. Quality Function Deployment Implementation in Construction: A Systematic Literature Review. Frontiers of Engineering Management, v. 3, n. 3, p. 224, 2016.

EVANGELISTA, P. P. DE A.; COSTA, D. B.; ZANTA, V. M. Alternativa sustentável para destinação de resíduos de construção classe A: sistemática para reciclagem em canteiros de obras. Ambiente Construído, n. 71, p. 23-40, 2010.

FRAGA, M. F. Panorama da geração de resíduos da construção civil em Belo Horizonte: medidas de minimização com base em projeto e planejamento de obras. p. 75, 2006.

GALVÃO, T. F.; PEREIRA, M. G. Revisões sistemáticas da literatura: passos para sua elaboração. Epidemiologia e Serviços de Saúde, v. 23, n. 1, p. 183-184, 2014.

GEUS, M. G.; GARCIAS, C. M. Gestão de resíduos de construção em edificações de grande porte na cidade de Ponta Grossa. Revista de Engenharia e Tecnologia V. 6, No. 2, p.124-139, Ago/2014

GHAFOURIAN, K. et al. The 100 Most Cited Scientific Papers in Construction and Demolition Waste Management. Research Journal of Applied Sciences, Engineering and Technology, v. 13, n. 9, p. 741-749, 2016 b.

GHAFOURIAN, K. et al. Current status of the research on construction and demolition waste management. Indian Journal of Science and Technology, v. 9, n. 35, 2016a.

IPEA. Pesquisa sobre Pagamento por Serviços Ambientais Urbanos para Gestão de Resíduos Sólidos. Instituto de Pesquisa Econômica Aplicada - Relatório de Pesquisa, p. 66, 2012.

KITCHENHAM, B. Procedures for performing systematic reviews. Keele, UK, Keele University, v. 33, n. TR/SE-0401, p. 28, 2004.

LEVY, Y.; ELLIS, T. J. A systems approach to conduct an effective literature review in support of information systems research. Informing Science, v. 9, p. 181-211, 2006.

LEVY, S, M.; HELENE, P.R.L.. Reciclagem de entulhos na construção civil e a solução política e ecologicamente correta. In: Simpósio Brasileiro de Tecnologias de Argamassa, 1º, Goiânia, Brasil. Agosto 1995 Anais. Goiânia, PP 315-325 LEVY, S. M. Reciclagem do Entulho de Construção Civil para Utilização como Agregado de Argamassas e Concretos. São Paulo, 1997. 145 f. Dissertação (Mestrado em Engenharia Civil) - Escola Politécnica, Universidade de São Paulo, São Paulo, 1997.

LINDE K, WILLICH SN. How objective are systematic reviews? Differences between reviews on complementary medicine. J R Soc Med. 2003;96:17-22.

MESQUITA, A. S. G. Análise da geração de resíduos sólidos da construção civil em teresina, piauí. Holos, v. 2, p. 58$65,2012$.

MINISTÉRIO DO MEIO AMBIENTE. Manual para implantação de sistema de gestão de resíduos de construção civil em consórcios públicos. p. 63, 2010.

MORAND, F. G. Estudo Das Principais Aplicações De Resíduos De Obra Como Materiais De Construção. 2016.

MUIANGA, E. A. D.; GRANJA, A. D.; RUIZ, J. DE A. Desvios de custos e prazos em empreendimentos da construção civil: categorização e fatores de influência. Ambiente construído, v. 15, n. 1, p. 79-97, 2015.

SAMPAIO, R.; MANCINI, M. Estudos de revisão sistemática: um guia para síntese criteriosa da evidência científica. Revista Brasileira de Fisioterapia, v. 11, n. 1, p. 83-89, 2007.

SANTOS, F. F. DOS et al. Adequação Dos Municípios Do Estado Do Rio Grande Do Sul À Legislação De Gestão De Resíduos Da Construção Civil. p. 1-17, 2012.

SCREMIN, L. B.; CASTILHOS JUNIOR, A. B. DE; ROCHA, J. C. Sistema de apoio ao gerenciamento de resíduos de construção e demolição para municípios de pequeno porte. Engenharia Sanitaria e Ambiental, v. 19, n. 2, p. 203-206, 2014.

SILVA, M. B. D. L. E. Novos Materiais À Base De Resíduos De Construção E Demolição (Rcd) E Resíduos De Produção De Cal (Rpc) Para Uso Na Construção Civil. 2014.

TAM, V. W. Y. Economic comparison of concrete recycling: A case study approach. Resources, Conservation and Recycling, v. 52, n. 5, p. 821-828, 2008.

UNITED STATES ENVIRONMENTAL PROTECTION AGENCY — EPA. The national biennial RCRA hazardous waste report. 2007.

\section{ANEXO A}

Artigos analisados na execução da revisão bibliográfica sistemática:

ABDEL-HAY, A. S. Properties of recycled concrete aggregate under different curing conditions. HBRC Journal, p. 1$6,2015$. 
AGRELA, F. et al. Influence of the sulphate content of recycled aggregates on the properties of cement-treated granular materials using Sulphate-Resistant Portland Cement. Construction and Building Materials, v. 68, p. 127-134, 2014. AGRELA, F. et al. Limiting properties in the characterisation of mixed recycled aggregates for use in the manufacture of concrete. Construction and Building Materials, v. 25, n. 10, p. 3950-3955, 2011.

AHMED, A.; UGAI, K.; KAMEI, T. Investigation of recycled gypsum in conjunction with waste plastic trays for ground improvement. Construction and Building Materials, v. 25, n. 1, p. 208-217, 2011.

AKBARNEZHAD, A. et al. Recycling of geopolymer concrete. Construction and Building Materials, v. 101, p. 152$158,2015$.

ANASTASIOU, E.; GEORGIADIS FILIKAS, K.; STEFANIDOU, M. Utilization of fine recycled aggregates in concrete with fly ash and steel slag. Construction and Building Materials, v. 50, p. 154-161, 2014.

ANTONY, J.; NAIR, D. G. Potential of Construction and Demolished Wastes as Pozzolana Potential of Construction and Demolished Wastes as Pozzolana. Procedia Technology, v. 25, n. December, p. 194-200, 2016.

ARISHA, A. et al. Using Blends of Construction \& Demolition Waste Materials and Recycled Clay Masonry Brick in Pavement. Procedia Engineering, v. 143, n. Ictg, p. 1317-1324, 2016.

ARULRAJAH, A. et al. Modulus of rupture evaluation of cement stabilized recycled glass/recycled concrete aggregate blends. Construction and Building Materials, v. 84, p. 146-155, 2015.

ARULRAJAH, A. et al. Physical properties and shear strength responses of recycled construction and demolition materials in unbound pavement base/subbase applications. Construction and Building Materials, v. 58, p. 245-257, 2014.

ARULRAJAH, A. et al. Influence of class F fly ash and curing temperature on strength development of fly ash-recycled concrete aggregate blends. Construction and Building Materials, v. 127, p. 743-750, 2016.

AWOYERA, P. O. et al. Characterization of ceramic waste aggregate concrete. HBRC Journal, p. 1-6, 2016.

BEHERA, M. et al. Recycled aggregate from C\&D waste \& its use in concrete - A breakthrough towards sustainability in construction sector: A review. Construction and Building Materials, v. 68, p. 501-516, 2014.

BELTRÁN, M. G. et al. Effect of cement addition on the properties of recycled concretes to reach control concretes strengths. Journal of Cleaner Production, v. 79, p. 124-133, 2014.

BRAVO, M. et al. Durability performance of concrete with recycled aggregates from construction and demolition waste plants. Construction and Building Materials, v. 77, p. 357-369, 2015.

BRAVO, M. et al. Mechanical performance of concrete made with aggregates from construction and demolition waste recycling plants. Journal of Cleaner Production, v. 99, p. 59-74, 2015.

CABRAL, A. E. B. et al. Mechanical properties modeling of recycled aggregate concrete. Construction and Building Materials, v. 24, n. 4, p. 421-430, 2010.

CABRAL, A. E. B. et al. Performance estimation for concretes made with recycled aggregates of construction and demolition waste of some Brazilian cities. Materials Research, v. 15, n. 6, p. 1037-1046, 2012.

CABRERA-COVARRUBIAS, F. G. et al. Implementation of interaction diagram of the properties in fresh for mortars with ceramic aggregates. Periodica Polytechnica: Civil Engineering, v. 61, n. 2, p. 335-340, 2017.

CARNEIRO, J. A. et al. Compressive stress-strain behavior of steel fiber reinforced-recycled aggregate concrete. Cement and Concrete Composites, v. 46, p. 65-72, 2014.

CERNI, G.; CARDONE, F.; BOCCI, M. Permanent deformation behaviour of unbound recycled mixtures. Construction and Building Materials, v. 37, p. 573-580, 2012.

CHEN, Z. et al. Recycling and reuse of construction and demolition waste in concrete-filled steel tubes: A review. Construction and Building Materials, v. 126, p. 641-660, 2016.

CONTRERAS, M. et al. Recycling of construction and demolition waste for producing new construction material (Brazil case-study). Construction and Building Materials, v. 123, p. 594-600, 2016.

CORINALDESI, V.; MORICONI, G. Behaviour of cementitious mortars containing different kinds of recycled aggregate. Construction and Building Materials, v. 23, n. 1, p. 289-294, 2009.

CORINALDESI, V.; MORICONI, G. Recycling of rubble from building demolition for low-shrinkage concretes. Waste Management, v. 30, n. 4, p. 655-659, 2010.

COURARD, L.; MICHEL, F.; DELHEZ, P. Use of concrete road recycled aggregates for Roller Compacted Concrete. Construction and Building Materials, v. 24, n. 3, p. 390-395, 2010.

CREE, D.; GREEN, M.; NOUMOWÉ, A. Residual strength of concrete containing recycled materials after exposure to fire: A review. Construction and Building Materials, v. 45, p. 208-223, 2013.

CRISTELO, N.; VIEIRA, C. S.; DE LURDES LOPES, M. Geotechnical and Geoenvironmental Assessment of Recycled Construction and Demolition Waste for Road Embankments. Procedia Engineering, v. 143, n. Ictg, p. 51-58, 2016.

CUENCA-MOYANO, G. M. et al. Influence of pre-soaked recycled fine aggregate on the properties of masonry mortar. Construction and Building Materials, v. 70, p. 71-79, 2014. 
CUENCA-MOYANO, G. M. et al. Development of the life cycle inventory of masonry mortar made of natural and recycled aggregates. Journal of Cleaner Production, v. 140, p. 1272-1286, 2017.

DEBIEB, F. et al. Roller compacted concrete with contaminated recycled aggregates. Construction and Building Materials, v. 23, n. 11, p. 3382-3387, 2009.

DOMINGO-CABO, A. et al. Creep and shrinkage of recycled aggregate concrete. Construction and Building Materials, v. 23, n. 7, p. 2545-2553, 2009.

DOSHO, Y. Development of a sustainable concrete waste recycling system: Application of recycled aggregate concrete produced by aggregate replacing method. Journal of Advanced Concrete Technology, v. 5, n. 1, p. 27-42, 2007.

ELCHALAKANI, M.; ELGAALI, E. Sustainable Concrete made of Construction and Demolition Wastes using Recycled Wastewater in the UAE. Journal of Advanced Concrete Technology, v. 10, n. 3, p. 110-125, 2012.

ETXEBERRIA, M. et al. Use of recycled fine aggregates for Control Low Strength Materials (CLSMs) production. Construction and Building Materials, v. 44, p. 142-148, 2013.

FONSECA, N.; DE BRITO, J.; EVANGELISTA, L. The influence of curing conditions on the mechanical performance of concrete made with recycled concrete waste. Cement and Concrete Composites, v. 33, n. 6, p. 637-643, 2011.

GALVÍN, A. P. et al. Analysis of leaching procedures for environmental risk assessment of recycled aggregate use in unpaved roads. Construction and Building Materials, v. 40, p. 1207-1214, 2013.

GAO, D.; ZHANG, L.; NOKKEN, M. Mechanical behavior of recycled coarse aggregate concrete reinforced with steel fibers under direct shear. Cement and Concrete Composites, v. 79, p. 1-8, 2017.

GASTALDI, D. et al. An investigation on the recycling of hydrated cement from concrete demolition waste. Cement and Concrete Composites, v. 61, p. 29-35, 2015.

GODINHO-CASTRO, A. P. et al. Incorporation of gypsum waste in ceramic block production: Proposal for a minimal battery of tests to evaluate technical and environmental viability of this recycling process. Waste Management, v. $32, \mathrm{n}$. 1, p. 153-157, 2012.

GÓMEZ-MEIJIDE, B. et al. Stiffness of cold asphalt mixtures with recycled aggregates from construction and demolition waste. Construction and Building Materials, v. 77, p. 168-178, 2015.

GÓMEZ-MEIJIDE, B.; PÉREZ, I. Binder-aggregate adhesion and resistance to permanent deformation of bitumenemulsion-stabilized materials made with construction and demolition waste aggregates. Journal of Cleaner Production, v. 129, p. 125-133, 2016.

GÓMEZ-MEIJIDE, B.; PÉREZ, I.; PASANDÍN, A. R. Recycled construction and demolition waste in Cold Asphalt Mixtures: Evolutionary properties. Journal of Cleaner Production, v. 112, p. 588-598, 2016.

GRDIC, Z. J. et al. Properties of self-compacting concrete prepared with coarse recycled concrete aggregate. Construction and Building Materials, v. 24, n. 7, p. 1129-1133, 2010.

HUDA, S. B.; ALAM, M. S. Mechanical behavior of three generations of $100 \%$ repeated recycled coarse aggregate concrete. Construction and Building Materials, v. 65, p. 574-582, 2014.

HUUHKA, S. et al. Reusing concrete panels from buildings for building: Potential in Finnish 1970s mass housing. Resources, Conservation and Recycling, v. 101, p. 105-121, 2015.

JAYASINGHE, C.; FONSEKA, W. M. C. D. J.; ABEYGUNAWARDHENE, Y. M. Load bearing properties of composite masonry constructed with recycled building demolition waste and cement stabilized rammed earth. Construction and Building Materials, v. 102, p. 471-477, 2016.

JIMÉNEZ, J. R. et al. Use of mixed recycled aggregates with a low embodied energy from non-selected CDW in unpaved rural roads. Construction and Building Materials, v. 34, p. 34-43, 2012.

JIMÉNEZ, J. R. et al. Use of fine recycled aggregates from ceramic waste in masonry mortar manufacturing. Construction and Building Materials, v. 40, p. 679-690, 2013.

JO, B.-W.; PARK, S.-K.; PARK, J.-C. Mechanical properties of polymer concrete made with recycled PET and recycled concrete aggregates. Construction and Building Materials, v. 22, n. 12, p. 2281-2291, 2008.

KABIR, S.; AL-SHAYEB, A.; KHAN, I. M. Recycled Construction Debris as Concrete Aggregate for Sustainable Construction Materials. Procedia Engineering, v. 145, p. 1518-1525, 2016.

KANELlOPOUlOS, A.; NICOLAIDES, D.; PETROU, M. F. Mechanical and durability properties of concretes containing recycled lime powder and recycled aggregates. Construction and Building Materials, v. 53, p. 253-259, 2014.

KOPER, A.; KOPER, W.; KOPER, M. Influence of Raw Concrete Material Quality on Selected Properties of Recycled Concrete Aggregates. Procedia Engineering, v. 172, p. 536-543, 2017.

KOU, S. C.; ZHAN, B. J.; POON, C. S. Feasibility study of using recycled fresh concrete waste as coarse aggregates in concrete. Construction and Building Materials, v. 28, n. 1, p. 549-556, 2012.

KOWALSKI, K. J. et al. Eco-friendly Materials for a New Concept of Asphalt Pavement. Transportation Research Procedia, v. 14, p. 3582-3591, 2016. 
KUMAR, R. Influence of recycled coarse aggregate derived from construction and demolition waste (CDW) on abrasion resistance of pavement concrete. Construction and Building Materials, v. 142, p. 248-255, 2017.

LÓPEZ GAYARRE, F. et al. Manufacture of concrete kerbs and floor blocks with recycled aggregate from C\&DW. Construction and Building Materials, v. 40, p. 1193-1199, 2013.

LÓPEZ GAYARRE, F. et al. Life cycle assessment for concrete kerbs manufactured with recycled aggregates. Journal of Cleaner Production, v. 113, p. 41-53, 2016.

LARBI, B.; ABDELAZIZ, M.; MILOUD, B. Study of the physico-mechanical properties of a recycled concrete incorporating admixtures by the means of NDT methods. Procedia Engineering, v. 108, p. 80-92, 2015.

LEDESMA, E. F. et al. Maximum feasible use of recycled sand from construction and demolition waste for eco-mortar production - Part-I: Ceramic masonry waste. Journal of Cleaner Production, v. 87, n. 1, p. 692-706, 2015.

LEITE, F. D. C. et al. Laboratory evaluation of recycled construction and demolition waste for pavements. Construction and Building Materials, v. 25, n. 6, p. 2972-2979, 2011.

LETELIER, V.; TARELA, E.; MORICONI, G. Mechanical Properties of Concretes with Recycled Aggregates and Waste Brick Powder as Cement Replacement. Procedia Engineering, v. 171, p. 627-632, 2017.

LIU, Q. et al. Investigation of using hybrid recycled powder from demolished concrete solids and clay bricks as a pozzolanic supplement for cement. Construction and Building Materials, v. 73, p. 754-763, 2014.

LÓPEZ-GAYARRE, F. et al. Assessment of properties of recycled concrete by means of a highly fractioned factorial design of experiment. Construction and Building Materials, v. 25, n. 10, p. 3802-3809, 2011.

LOTFI, S. et al. Performance of recycled aggregate concrete based on a new concrete recycling technology. Construction and Building Materials, v. 95, p. 243-256, 2015.

MARTÍN-MORALES, M. et al. Effect of recycled aggregate on physical-mechanical properties and durability of vibrocompacted dry-mixed concrete hollow blocks. Construction and Building Materials, v. 145, p. 303-310, 2017.

MARTÍN-MORALES, M. et al. Characterization of recycled aggregates construction and demolition waste for concrete production following the Spanish Structural Concrete Code EHE-08. Construction and Building Materials, v. 25, n. 2, p. 742-748, 2011.

MAS, B. et al. Influence of the amount of mixed recycled aggregates on the properties of concrete for non-structural use. Construction and Building Materials, v. 27, n. 1, p. 612-622, 2012.

MATAR, P.; EL DALATI, R. Strength of masonry blocks made with recycled concrete aggregates. Physics Procedia, v. 21, p. 180-186, 2011.

MATIAS, D. et al. Mechanical properties of concrete produced with recycled coarse aggregates - Influence of the use of superplasticizers. Construction and Building Materials, v. 44, p. 101-109, 2013.

MEDINA, C. et al. Gas permeability in concrete containing recycled ceramic sanitary ware aggregate. Construction and Building Materials, v. 37, p. 597-605, 2012.

MEDINA, C. et al. Effect of the constituents (asphalt, clay materials, floating particles and fines) of construction and demolition waste on the properties of recycled concretes. Construction and Building Materials, v. 79, p. 22-33, 2015. MEDINA, C. et al. Influence of mixed recycled aggregate on the physical-mechanical properties of recycled concrete. Journal of Cleaner Production, v. 68, p. 216-225, 2014.

MEDINA, C.; FRÍAS, M.; SÁNCHEZ DE ROJAS, M. I. Leaching in concretes containing recycled ceramic aggregate from the sanitary ware industry. Journal of Cleaner Production, v. 66, p. 85-91, 2014.

MORICONI, G.; TARELA, E. Combined effects of recycled hydrated cement and recycled aggregates on the mechanical properties of concrete. Construction and Building Materials, v. 132, p. 365-375, 2017.

OLIVEIRA, R.; DE BRITO, J.; VEIGA, R. Reduction of the cement content in rendering mortars with fine glass aggregates. Journal of Cleaner Production, v. 95, p. 75-88, 2015.

OSSA, A.; GARC??A, J. L.; BOTERO, E. Use of recycled construction and demolition waste (CDW) aggregates: A sustainable alternative for the pavement construction industry. Journal of Cleaner Production, v. 135, p. 379-386, 2016. ÖZALP, F. et al. Effects of recycled aggregates from construction and demolition wastes on mechanical and permeability properties of paving stone, kerb and concrete pipes. Construction and Building Materials, v. 110, p. 17-23, 2016.

PARTHIBAN, K.; SARAVANA RAJA MOHAN, K. Influence of recycled concrete aggregates on the engineering and durability properties of alkali activated slag concrete. Construction and Building Materials, v. 133, p. 65-72, 2017.

PASANDÍN, A. R.; PÉREZ, I. Effect of ageing time on properties of hot-mix asphalt containing recycled concrete aggregates. Construction and Building Materials, v. 52, p. 284-293, 2014.

PASANDÍN, A. R.; PÉREZ, I. Laboratory evaluation of hot-mix asphalt containing construction and demolition waste. Construction and Building Materials, v. 43, p. 497-505, 2013.

PÉREZ, I.; PASANDÍN, A. R.; MEDINA, L. Hot mix asphalt using C\&D waste as coarse aggregates. Materials and Design, v. 36, p. 840-846, 2012.

PETRILlO, A. et al. Eco-sustainable Geopolymer Concrete Blocks Production Process. Agriculture and Agricultural Science Procedia, v. 8, p. 408-418, 2016. 
PIÑEIRO, S. R.; DEL R??O MERINO, M.; P??REZ GARC??A, C. New Plaster Composite with Mineral Wool Fibres from CDW Recycling. Advances in Materials Science and Engineering, v. 2015, 2015.

PUERTAS, F. et al. Alkali-activated cement mortars containing recycled clay-based construction and demolition waste. Ceramics - Silikaty, v. 59, n. 3, p. 202-210, 2015.

RAHMAN, M. A. et al. Suitability of recycled construction and demolition aggregates as alternative pipe backfilling materials. Journal of Cleaner Production, v. 66, p. 75-84, 2014.

RAO, A.; JHA, K. N.; MISRA, S. Use of aggregates from recycled construction and demolition waste in concrete. Resources, Conservation and Recycling, v. 50, n. 1, p. 71-81, 2007.

REIG, L. et al. Properties and microstructure of alkali-activated red clay brick waste. Construction and Building Materials, v. 43, p. 98-106, 2013.

RICHARDSON, A.; COVENTRY, K.; BACON, J. Freeze/thaw durability of concrete with recycled demolition aggregate compared to virgin aggregate concrete. Journal of Cleaner Production, v. 19, n. 2-3, p. 272-277, 2011.

ROQUE, A. J. et al. Recycling of CDW and Steel Slag in Drainage Layers of Transport Infrastructures. Procedia Engineering, v. 143, n. Ictg, p. 196-203, 2016.

ROQUE, A. J. et al. Assessment of Environmental Hazardous of Construction and Demolition Recycled Materials (C\&DRM) from Laboratory and Field Leaching Tests Application in Road Pavement Layers. Procedia Engineering, v. 143, n. Ictg, p. 204-211, 2016.

SABAI, M. M. et al. Concrete block production from construction and demolition waste in Tanzania. Resources, Conservation and Recycling, v. 72, p. 9-19, 2013.

SAIZ MARTÍNEZ, P. et al. Comparative study of three types of fine recycled aggregates from construction and demolition waste (CDW), and their use in masonry mortar fabrication. Journal of Cleaner Production, v. 118, p. 162$169,2016$.

SÁNCHEZ-ROLDÁN, Z. et al. Study of potential advantages of pre-soaking on the properties of pre-cast concrete made with recycled coarse aggregate. Materiales de Construccion, v. 66, n. 321, 2016.

SARAVANAKUMAR, P.; ABHIRAM, K.; MANOJ, B. Properties of treated recycled aggregates and its influence on concrete strength characteristics. Construction and Building Materials, v. 111, p. 611-617, 2016.

SEGANTINI, A. A. DA S.; WADA, P. H. An evaluation of the composition of soil cement bricks with construction and demolition waste/Estudo de dosagem de tijolos de solo-cimento com adição de resíduos de construção e demolição. Acta Scientiarum - Technology, v. 33, n. 2, p. 179-183, 2011.

SENARATNE, S. et al. The costs and benefits of combining recycled aggregate with steel fibres as a sustainable, structural material. Journal of Cleaner Production, v. 112, p. 2318-2327, 2016.

SERPA, D. et al. ASR of mortars containing glass. Construction and Building Materials, v. 47, p. 489-495, 2013.

SHAHIDAN, S. et al. Utilizing Construction and Demolition (C\&D) Waste as Recycled Aggregates (RA) in Concrete. Procedia Engineering, v. 174, p. 1028-1035, 2017.

SHAIKH, F. U. A. Mechanical and durability properties of fly ash geopolymer concrete containing recycled coarse aggregates. International Journal of Sustainable Built Environment, v. 5, n. 2, p. 277-287, 2016.

SILVA, R. V.; DE BRITO, J.; DHIR, R. K. Performance of cementitious renderings and masonry mortars containing recycled aggregates from construction and demolition wastes. Construction and Building Materials, v. 105, p. 400$415,2016$.

SILVA, R. V.; DE BRITO, J.; DHIR, R. K. Prediction of the shrinkage behavior of recycled aggregate concrete: A review. Construction and Building Materials, v. 77, p. 327-339, 2015.

SILVA, R. V.; DE BRITO, J.; DHIR, R. K. Properties and composition of recycled aggregates from construction and demolition waste suitable for concrete production. Construction and Building Materials, v. 65, p. $201-217$, 2014.

SILVA, R. V.; DE BRITO, J.; DHIR, R. K. Tensile strength behaviour of recycled aggregate concrete. Construction and Building Materials, v. 83, p. 108-118, 2015.

SILVA, Y. F. et al. Properties of self-compacting concrete on fresh and hardened with residue of masonry and recycled concrete. Construction and Building Materials, v. 124, p. 639-644, 2016.

SOUTSOS, M. N.; TANG, K.; MILLARD, S. G. Concrete building blocks made with recycled demolition aggregate. Construction and Building Materials, v. 25, n. 2, p. 726-735, 2011.

SOUTSOS, M. N.; TANG, K.; MILLARD, S. G. The use of recycled demolition aggregate in precast concrete products - Phase III: Concrete pavement flags. Construction and Building Materials, v. 36, p. 674-680, 2012.

SOUTSOS, M. N.; TANG, K.; MILLARD, S. G. Use of recycled demolition aggregate in precast products, phase II: Concrete paving blocks. Construction and Building Materials, v. 25, n. 7, p. 3131-3143, 2011.

SUÁREZ, S.; ROCA, X.; GASSO, S. Product-specific life cycle assessment of recycled gypsum as a replacement for natural gypsum in ordinary Portland cement: Application to the Spanish context. Journal of Cleaner Production, v. 117, p. $150-159,2016$. 
SURYA, M.; VVL, K. R.; LAKSHMY, P. Recycled Aggregate Concrete for Transportation Infrastructure. Procedia Social and Behavioral Sciences, v. 104, n. m, p. 1158-1167, 2013.

TABSH, S. W.; ABDELFATAH, A. S. Influence of recycled concrete aggregates on strength properties of concrete. Construction and Building Materials, v. 23, n. 2, p. 1163-1167, 2009.

TAM, V. W. Y.; WANG, K. TAM, C. M. Ways to facilitate the use of recycled aggregate concrete. Proceedings Of The Institution Of Civil Engineers, n. WR3, p. 125-129, 2007.

THOMAS, C. et al. Influence of recycled aggregates containing sulphur on properties of recycled aggregate mortar and concrete. Composites Part B: Engineering, v. 45, n. 1, p. 474-485, 2013.

ULSEN, C. et al. Production of recycled sand from construction and demolition waste. Construction and Building Materials, v. 40, p. 1168-1173, 2013.

VÄNTSI, O.; KÄRKI, T. Utilization of recycled mineral wool as filler in wood-polypropylene composites. Construction and Building Materials, v. 55, p. 220-226, 2014.

VIEIRA, C. S.; PEREIRA, P. M.; LOPES, M. D. L. Recycled Construction and Demolition Wastes as filling material for geosynthetic reinforced structures. Interface properties. Journal of Cleaner Production, v. 124, p. 299-311, 2016.

VIEIRA, T. et al. Durability-related performance of concrete containing fine recycled aggregates from crushed bricks and sanitary ware. Materials and Design, v. 90, p. 767-776, 2016.

WAGIH, A. M. et al. Recycled construction and demolition concrete waste as aggregate for structural concrete. HBRC Journal, v. 9, n. 3, p. 193-200, 2013.

XIAO, Z. et al. Properties of partition wall blocks prepared with high percentages of recycled clay brick after exposure to elevated temperatures. Construction and Building Materials, v. 49, p. 56-61, 2013.

XUAN, D. X.; MOLENAAR, A. A. A.; HOUBEN, L. J. M. Deformation behavior of cement treated demolition waste with recycled masonry and concrete subjected to drying and temperature change. Cement and Concrete Composites, v. 68 , p. 37-34, 2016.

XUAN, D. X. et al. Influence of quality and variation of recycled masonry aggregates on failure behavior of cement treated demolition waste. Construction and Building Materials, v. 71, p. 521-527, 2014.

XUAN, D. X.; MOLENAAR, A. A. A.; HOUBEN, L. J. M. Evaluation of cement treatment of reclaimed construction and demolition waste as road bases. Journal of Cleaner Production, v. 100, p. 77-83, 2015.

YANG, J.; DU, Q.; BAO, Y. Concrete with recycled concrete aggregate and crushed clay bricks. Construction and Building Materials, v. 25, n. 4, p. 1935-1945, 2011.

YOUNIS, K. H.; PILAKOUTAS, K. Strength prediction model and methods for improving recycled aggregate concrete. Construction and Building Materials, v. 49, p. 688-701, 2013.

ZEGA, C. J.; DI MAIO, Á. A. Use of recycled fine aggregate in concretes with durable requirements. Waste Management, v. 31, n. 11, p. 2336-2340, 2011.

ZHU, P. et al. Investigation of using recycled powder from waste of clay bricks and cement solids in reactive powder concrete. Construction and Building Materials, v. 113, p. 246-254, 2016.

ZONG, L.; FEI, Z.; ZHANG, S. Permeability of recycled aggregate concrete containing fly ash and clay brick waste. Journal of Cleaner Production, v. 70, p. 175-182, 2014. 